\title{
Cryosurgery to remove perichondrium for the rabbit ear hypertrophic scar model: a simplified method
}

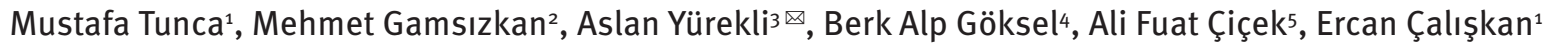

\begin{abstract}
Introduction: The rabbit ear hypertrophic scar model is a preferred animal model of excessive scarring for investigating the scarring process and novel treatment modalities. In this model, surgical removal of perichondrium can be challenging, and it is often insufficient or damages the underlying cartilage. It is hypothesized that cryosurgery would offer a more efficient alternative to conventional surgery. The objective of this study was to compare structural changes in scar tissues in two groups of the hypertrophic scar model: cryosurgery compared to standard conventional surgery.

Methods: We introduced a novel technique to remove perichondrium using cryosurgery. Hypertrophic scars obtained with conventional surgery and cryosurgery were studied in a left-right comparison method. Comparative parameters included the histological structure of the scars and structural changes in the cartilage just beneath the scarring.

Results: Cryosurgery produced similar scars in comparison to conventional surgery. Although statistically not significant $(p=$ 0.16), the histological findings of cartilage damage were lower in the cryosurgery group (six out of 21) compared to the established model (10 out of 20$)$.

Conclusions: This study suggests that cryotherapy can be used for removal of perichondrium.
\end{abstract}

Keywords: animal model, cryotherapy, hypertrophic scar

Received: 2 September 2018 | Returned for modification: 16 November 2018 | Accepted: 19 January 2019

\section{Introduction}

The rabbit ear hypertrophic scar model (REHSM) is one of the most advanced animal models of excessive scarring because it provides reproducible scars similar to human scarring with regard to their macroscopic and histological appearance $(1,2)$. These scars persist for months and respond to scar-modulating treatments with various agents (1-4). The model is based on the removal of skin and underlying perichondrium at least $7 \mathrm{~mm}$ in diameter using a punch biopsy with a corresponding size (Fig. 1a-b). Complete removal of the perichondrial layer is necessary because it delays reepithelialization for 8 to 14 days in $7 \mathrm{~mm}$ punch biopsy wounds (2). Perichondrial tissues remaining at the wound bed lead to faster epithelialization, and so an optimal hypertrophic scar (HS) may not form. Practically, if the perichondrium cannot be removed thoroughly at once, removal of the residual perichondrium can be problematic. During the removal of perichondrial remnants, the underlying cartilaginous tissue can be damaged (Fig. 1c) even by experienced hands. Jia et al. previously reported that the surgical removal of perichondrium could result in cartilage desiccation, although they had been implementing REHSM with success for over a decade (5). In this case, injury-related changes can occur under the scar tissue, including cartilaginous proliferation and even metaplastic changes. Even though they can be detected histologically, the existence of these tissues can lead to misperceptions of scar height macroscopically or affect the therapeutic responses of scar-modulating agents.

Due to these reasons, we looked for an alternate approach to remove perichondrium. As an efficient and well-controlled tool targeting tissue removal, cryosurgery is being performed by many dermatologists in their everyday practice (6). In this study, we hypothesized whether cryosurgery could be used effectively for the removal of perichondrium in a simpler and easily reproducible model instead of the previously described conventional surgery.

\section{Methods}

The study was carried out with the approval of the Animal Experimentation Ethics Committee of our institution (no. 14/190). Six young male New Zealand white rabbits (3,000 to 3,500 g) were used. The animals were kept under standardized housing and animal care conditions following the guidelines of the ethics committee.

\section{Surgical procedure}

Removal of the perichondrium was performed with conventional surgery on the left ears and cryosurgery was performed on the right ears. To prevent any possible performance bias, surgical removal was performed by an experienced surgeon as previously
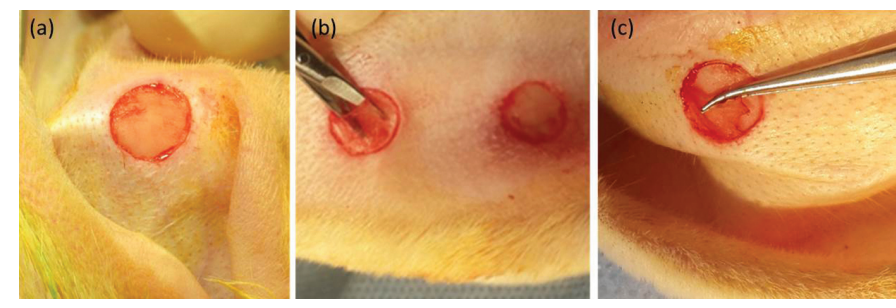

Figure 1 | Appearance of the wounds on the rabbit ear after the removal of a) skin and b) perichondrium; c) cartilage damage on the right side of the wound and residual perichondrium on the left side (tip of the instrument).

12Department of Dermatology, Gülhane Medical Faculty, University of Health Sciences, Ankara, Turkey. ${ }^{2}$ Department of Pathology, Düzce University, Düzce, Turkey. ${ }^{3}$ Bayburt State Hospital, Bayburt, Turkey. ${ }^{4}$ üulhane Department of Animal Experimentation, Institute of Health Sciences, University of Health Sciences, Ankara Turkey. ${ }^{5}$ Department of Pathology, Gülhane Medical Faculty, University of Health Sciences, Ankara, Turkey. $\bowtie$ Corresponding author: aslanyureklio3@hotmail.com 
described $(1,2)$. Cryosurgery was performed by the investigating dermatologist (Fig. 2).
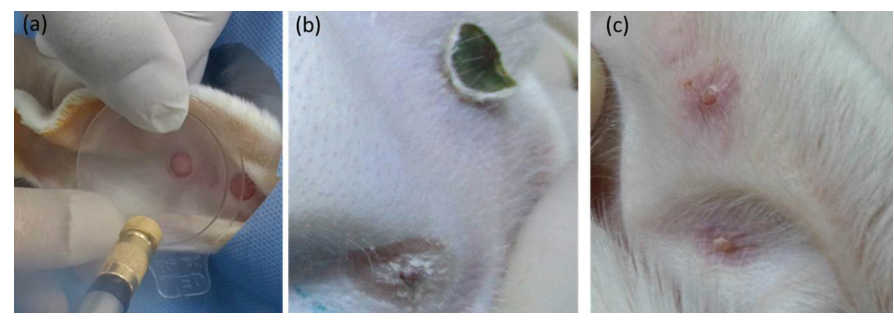

Figure 2 a) Application of liquid nitrogen to perichondrium and b) desquamation of the necrotic perichondrial layer on day $16 ; c)$ appearance of fully evolved hypertrophic scars with cryosurgery on day 28.

The rabbits were anesthetized with an intramuscular injection of ketamine $(45 \mathrm{mg} / \mathrm{kg})$ and xylazine $(5 \mathrm{mg} / \mathrm{kg})$. Four wounds were created on each ear with an $8 \mathrm{~mm}$ biopsy punch on the ventral skins of the ears. After the removal of the skin, perichondrial tissues on the right ears were subjected to liquid nitrogen with a cryospray (spray tip C) from an approximately $5 \mathrm{~cm}$ distance thrice for 2 to 3 seconds ( 3 seconds for proximal parts, and 2 seconds for distal parts of the ear because of its thinner structure). At the end of the procedure, 48 wounds were created on six rabbits. On day 28 , scars were harvested with more than a $5 \mathrm{~mm}$ margin of adjacent skin and the animals were sacrificed.

\section{Histological evaluation}

Histological evaluation was performed by two pathologists, who were blinded to the origin of the samples. After fixation with $10 \%$ buffered formaldehyde solution, the samples were put into a buffered formic acid solution for decalcification. Once processed, the samples were embedded in paraffin. We obtained $5 \mu \mathrm{m}$ sections from the samples and stained them with hematoxylin-eosin for routine histopathological evaluation, and Masson-trichrome stain for morphometric analysis of scar tissue.

The histological evaluations focused on the HS tissue itself to determine the efficacies of the techniques and on the area under the scar to identify injury-related findings. For the morphometric analysis of the scar tissue, the hypertrophic index (HI), fibroblast density, and collagen index (CI) were used, as previously described in detail (7). Briefly, $\mathrm{HI}$ and CI were calculated as the ratio of the greatest vertical height and collagen density of scar tissue to adjacent unwounded tissue around the scar. The fibroblast density was the number of fibroblasts within the scar in $1 \mathrm{~mm}$ (2).

Structural changes under the scar were classified according to predominant tissue type (Fig. 3). Cartilaginous tissue, whether hypertrophic or not, was considered normal, whereas osseous and fibrous tissue replacements were considered abnormal reaction patterns.

\section{Statistical analysis}

The Statistical Package for Social Sciences (SPSS Inc, Chicago, IL, USA) version 20.0 was used to perform all the statistical analyses. The Kolmogorov-Smirnov test was used for analyzing the distribution pattern of the data, and normally distributed continuous variables were expressed as mean \pm standard deviation. Mean values between groups were analyzed using Student's t-test. Differences were considered significant at $p<0.05$.
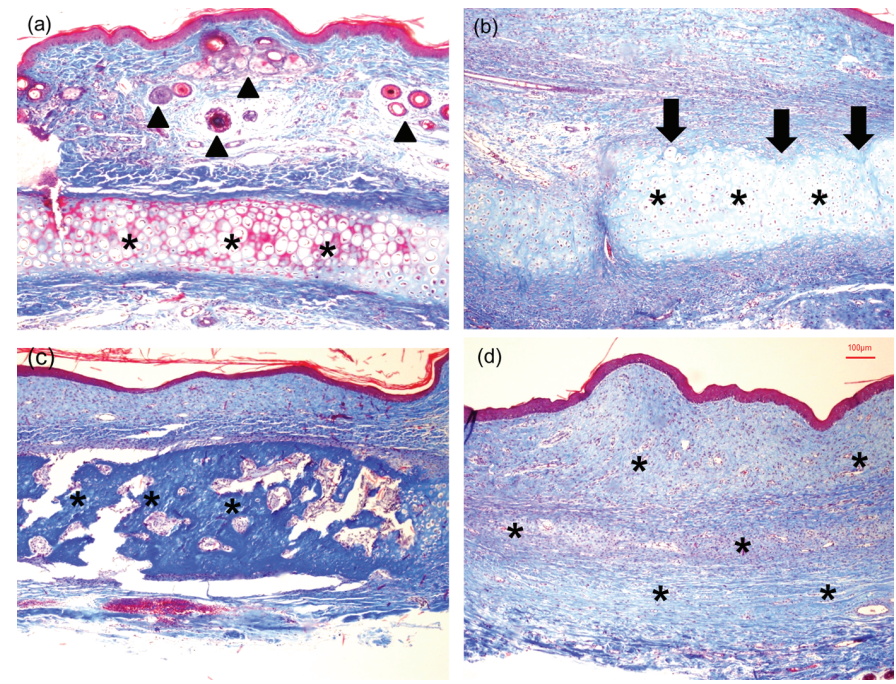

Figure 3 | Histological structure of dermis and cartilage, displaying a) normal dermis including skin appendages (arrow heads) and normal cartilage (asterisks); b) hypertrophic bluish cartilage (asterisks) due to loss of intercellular chondroid purple-lilac matrix, it also has an irregular boundary line (arrows) compared to normal histology, there are no skin appendages; c) metaplastic bone tissue (asterisks); and d) completely cellular fibrous tissue (asterisks) and slight increase of vascularity (Masson's trichrome stain, $\times 100$ ).

\section{Results}

Four wounds in the surgical excision group and three wounds in the cryosurgery group were eliminated from morphometric analysis due to invagination of epithelial tissues, most probably due to improper technique. All other wounds exhibiting clinical features compatible with HSs were included in the histological evaluation. Perichondrial tissue was not evident in fully evolved HSs, but it was clearly observed in ambiguous scars and metaplastic tissues.

We found no statistically significant difference between the two methods in relation to scar morphology (Table 1). Based on the similar results of scar morphology with each method, cryosurgery is as efficient as conventional surgery for causing HS formation. With regard to the structure of the tissue under the scar (Fig. 3), cryosurgery yielded a higher rate of normal tissue in comparison to the conventional surgery group: 15 out of 20 (71.5\%) versus 10 out of $20(50 \% ; p=0.16$; Table 2$)$. However the difference between groups did not reach the level of statistical significance $(p=0.16)$.

In both groups, in addition to the loss of skin appendageal structures, irregular, hypercellular, hyalinized collagen band fibers and a slight increase in vascularity were seen in the dermis of the samples compared to normal histology.

Table 1 | Comparison of histological parameters of scar tissues in treatment groups.

\begin{tabular}{lccc}
\hline Parameter & $\begin{array}{c}\text { Excisional surgery } \\
(n=20)\end{array}$ & $\begin{array}{c}\text { Cryosurgery } \\
(n=21)\end{array}$ & $p$-value \\
\hline Hypertrophic index & $1.52 \pm 0.4$ & $1.63 \pm 0.5$ & 0.459 \\
Collagen index & $1.2 \pm 0.1$ & $1.1 \pm 0.1$ & 0.076 \\
Fibroblast count $\left(\mathrm{mm}^{2}\right)$ & $631.4 \pm 197.7$ & $661.9 \pm 184.4$ & 0.612 \\
\hline
\end{tabular}

All values are given as mean \pm standard deviation. The Kolmogorov-Smirnov test was used for analysis.

Table 2 | Comparison of histological structure under scarring.

\begin{tabular}{lccc}
\hline Predominant tissue & $\begin{array}{c}\text { Excisional surgery } \\
(n=20)\end{array}$ & $\begin{array}{c}\text { Cryosurgery } \\
(n=21)\end{array}$ & $p$-value \\
\hline Cartilage & 10 & 15 & 0.16 \\
$\begin{array}{l}\text { Fibrous/metaplastic } \\
\text { bone tissue }\end{array}$ & 10 & 6 & \\
\hline
\end{tabular}

Student's $t$-test was used for analysis. 


\section{Discussion}

This preliminary study compared morphological parameters within scar tissue to evaluate the efficacy of the method and the morphological features of the cartilage under the scar to reveal any collateral injury that can affect the success of REHSM.

The histological parameters of cryosurgery within the scar revealed high similarity to conventional surgery without statistical significance. In this regard, it can be said that cryosurgery is capable of developing consistent HSs, parallel to the previously described conventional method.

Despite avoidance during the procedure, cartilage can be injured when interfering with perichondrium. Cartilage proliferation is an anticipated finding and has been reported previously with this model $(1,2)$. However, considering the osseous and fibrous tissues determined in this study, we were unable to find any previous reports with REHSM in our PubMed search. Interestingly, in 1961, Joseph et al. investigated structural changes of the rabbit ear during wound healing after the removal of skin only or in combination with cartilage, concluding that metaplastic bone formation was detected in all the samples of both groups (8). Replacement of cartilage with fibrous tissue was also defined in rabbit ears as a result of full-thickness cartilage injury (9). There exist numerous cases of localized bone tissue formation in humans, mostly associated with local injury and intense inflammatory reaction (10). Therefore, we considered metaplastic tissues under the scar in response to tissue injury. In our study, metaplastic bone tissue was detected in 30\% and 19\%, and fibrous tissue in $20 \%$ and $9.5 \%$, of the wounds in excisional surgery and cryosurgery groups, respectively. Although statistically insignificant, these outcomes indicate the superiority of cryosurgery over excisional surgery with regard to cartilage injury.

Limited damage to cartilage with cryosurgery can be attributed to its adjustability, leading to well-aimed and controlled destruction of target tissue. It is possible to change freezing parameters such as duration of freezing, repetition of freeze-thaw cycles, and probe size to maximize the destruction of target tissue while minimizing the injury to adjacent non-targeted tissue. Another possible explanation may depend on the avascular structure of cartilage. As is known, the destructive mechanisms induced by cryosurgery are not limited to its physical effects (11). In the vascular phase of cryoreaction, delayed-type cell destruction occurs due to vascular stasis and subsequently ischemic necrosis. However, as an avascular tissue, the vascular phase cannot occur within cartilage and can therefore make it resistant to cryosurgery in our case.

This study had some limitations. It was carried out on a relatively small number of samples and a larger number of samples could help draw more accurate conclusions about some differences that did not reach a statistically significant level in this study.

\section{Conclusions}

In conclusion, the susceptibility of perichondrium to cold injury compared to cartilage, coupled with the versatility of cryosurgery, which enables selective damage to the targeted tissue, makes this procedure suitable for the delicate removal of perichondrium. Hence, cryosurgery, as an efficient procedure compared to conventional surgery, can be considered a useful alternative for REHSM, lessening limitations including cartilage injury and the need for skilled surgeons.

\section{Acknowledgement}

This study was financially supported by the Gülhane School of Medicine Research Grant committee and was conducted at the Animal Research Laboratory of the same institution.

\section{References}

1. Morris DE, Wu L, Zhao LL, Bolton L, Roth SI, Ladin DA, et al. Acute and chronic animal models for excessive dermal scarring: quantitative studies. Plast Reconstr Surg. 1997;100:674-81.

2. Kloeters O, Tandara A, Mustoe TA. Hypertrophic scar model in the rabbit ear: a reproducible model for studying scar tissue behavior with new observations on silicone gel sheeting for scar reduction. Wound Repair Regen. 2007;15:40-5.

3. Ramos ML, Gragnani A, Ferreira LM. Is there an ideal animal model to study hypertrophic scarring? J Burn Care Res. 2008;29:363-8.

4. Huang YC, Huang $\mathrm{CT}$, Hu C, Wong TW. Portable ultraviolet light $\mathrm{A} 1$ light source to treat hypertrophic scar. Dermatologica Sinica. 2015;33:11-5.

5. Jia S, Zhao Y, Mustoe TA. The effects of topically applied silicone gel and its silver derivative on the prevention of hypertrophic scarring in two rabbit ear-scarring models. J Plast Reconstr Aesthet Surg. 2011;64:332-4.

6. Zouboulis CC. Principles of cutaneous cryosurgery: an update. Dermatology. 1999;198:111-7.
7. Çalıskan E, Gamsızkan M, Açıkgöz G, Durmuş M, Toklu S, Doğrul A, et al. Intralesional treatments for hypertrophic scars: comparison among corticosteroid, 5 -fluorouracil and botulinum toxin in rabbit ear hypertrophic scar model. Eur Rev Med Pharmacol Sci. 2016;20:1603-8.

8. Joseph J, Thomas GA, Tynen J. The reaction of the ear cartilage of the rabbit and guinea-pig to trauma. J Anat. 1961;95:56.

9. Chlebicki CA, Protsenko DE, Wong BJ. Preliminary investigations on therapy thresholds for laser dosimetry, cryogen spray cooling duration, and treatment cycles for laser cartilage reshaping in the New Zealand white rabbit auricle. Lasers Med Sci. 2014;29:1099-109.

10. Manni JJ, Berénos-Riley LC. Ossification of the external ear: a case report and review of the literature. Eur Arch Otorhinolaryngol. 2005;262:961-4.

11. Chua KJ, Chou SK, Ho JC. An analytical study on the thermal effects of cryosurgery on selective cell destruction. J Biomech. 2007;40:100-16. 